\title{
Active buckling control of a beam-column with circular cross-section using piezo-elastic supports and integral LQR control
}

\author{
Maximilian Schaeffner ${ }^{1}$, Benedict Götz ${ }^{1}$ and Roland Platz ${ }^{2}$ \\ ${ }^{1}$ System Reliability and Machine Acoustics SzM, Technische Universität Darmstadt, \\ Magdalenenstraße 4, D-64289 Darmstadt, Germany \\ ${ }^{2}$ Fraunhofer Institute for Structural Durability and System Reliability LBF, \\ Bartningstraße 47, D-64289 Darmstadt, Germany \\ E-mail: schaeffner@szm.tu-darmstadt.de \\ Article reference: SMS-102993, first submission 22-Dec-2015, revision 15-Apr-2016
}

\begin{abstract}
Buckling of slender beam-columns subject to axial compressive loads represents a critical design constraint for light-weight structures. Active buckling control provides a possibility to stabilize slender beam-columns by active lateral forces or bending moments. In this paper, the potential of active buckling control of an axially loaded beam-column with circular solid cross-section by piezo-elastic supports is investigated experimentally. In the piezo-elastic supports, lateral forces of piezoelectric stack actuators are transformed into bending moments acting in arbitrary directions at the beam-column ends. A mathematical model of the axially loaded beam-column is derived to design an integral linear quadratic regulator (LQR) that stabilizes the system. The effectiveness of the stabilization concept is investigated in an experimental test setup and compared with the uncontrolled system. With the proposed active buckling control it is possible to stabilize the beam-column in arbitrary lateral direction for axial loads up to the theoretical critical buckling load of the system.
\end{abstract}

PACS numbers: Mechanical systems, control of, 45.80.+r, Buckling static, 46.32.+x, Vibrations mechanical, 46.40.-f, Beams structural mechanics, 46.70.De

Keywords: active buckling control, piezo-elastic supports, integral LQR, beam-column

Submitted to: Smart Mater. Struct. 


\section{Introduction}

Buckling of slender and compressively loaded beam-columns is a critical failure mode in the design of light-weight structures. The theory of buckling for passive beam-columns has been thoroughly investigated, [1]. A general approach to passively increase the critical buckling load is to change the geometry, e. g. length and cross-section area, or the material so that the beam-column withstands higher loads. This, however, is sometimes not desirable because of given design constraints. In these cases, active buckling control without significant change in geometry and material provides a suitable approach to increase the maximum bearable load of a given structure. Active buckling control of slender beam-columns with rectangular cross-section and different boundary conditions has been investigated numerically and experimentally several times, [2, 3, 4, 5, 6, 7, 8, 9].

One way to compare the active approaches for the different investigated structures is to take into account the slenderness ratio $s=l_{\text {eff }} / i$ that is derived from the beam's effective buckling length $l_{\text {eff }}$ and the gyration radius $i$. Usually, elastic EuLER buckling may occur for slenderness ratios higher than the limiting slenderness ratio $s>s_{\lim }=\pi \sqrt{E / \sigma_{\mathrm{p}}}$ which is dependent on the material's Young's modulus $E$ and the proportional limit $\sigma_{\mathrm{p}}$, [1]. Often, surface bonded piezoelectric patches were applied to beam-columns with rectangular cross-section and relatively high slenderness ratios to induce active bending moments that counteract the deformation, $[2,3,4,5,6]$. In a numerical study, the buckling load of a pinned-pinned beam with piezoelectric patches along the entire length and slenderness ratio $s=530$ could be increased above the first critical buckling load by $280 \%$ for quasi-static load increase, [2]. In a similar numerical study with the same beam geometry and slenderness ratio with pinned-pinned boundary conditions and discrete piezo patches at two beam positions, an increase in the critical buckling load by $780 \%$ was achieved for the stabilization of a non-zero initial deformation, [3]. In an experimental investigation, actively controlled piezoelectric patches were applied to predeformed carbon-epoxy composite columns with slenderness ratio $s=970$ and the critical buckling load could be increased by $37 \%$ compared to the column without actively controlled patches and by $7 \%$ compared to the theoretical buckling load of the undeformed column, [4]. For a beam-column with $s=670$ and active piezoelectric patch actuators attached along the entire surface with additional stiffeners to cover gaps between the actuators, the buckling load of the passive system could be increased in an experimental study by $460 \%$, [5]. In a recent study, the buckling load of a fixed-free beam-column with slenderness ratio $s=1760$ and piezoelectric patches attached along the entire surface could be increased by $105 \%$ using proportional feedback control, [6]. In another stabilization concept, a predeformed beam-column with $s=300$ and eccentrically embedded shape memory alloys was investigated experimentally and an increase of $11 \%$ in the critical buckling load was achieved, [7].

The active stabilization concept investigated by earlier own studies [8,9] used piezoelectric stack actuators to apply active lateral forces near the base of a fixed-pinned 
beam-column with rectangular cross-section and slenderness ratio $s=725$. Compared to other studies mentioned above, most of the beam-column's surface was kept free from any actuator like piezoelectric patches, so the beam-column's stiffness and, respectively, the slenderness ratio was not influenced by additional actuators. Only strain gauges were applied to the surface. In [8, 9] and additional to all other studies, a lateral disturbance force representing uncertainty in the beam-column's loading was introduced to investigate the approach of active buckling control with lateral forces near the base of the fixed-pinned beam-column. In an experimental study, an increase in the critical axial buckling load of $40 \%$ was achieved by using a linear-quadratic regulator (LQR) to control the first three modes of the supercritically loaded beam-column, [9].

Active buckling control of beam-columns with circular cross-section has not been investigated so far. In [10], active buckling control of a circular beam-column with slenderness ratio $s=500$ and active lateral forces acting near the beam-column's fixed base was investigated and an increase of $110 \%$ in the critical buckling load was achieved in numerical simulations. The studies mentioned before investigated beam-columns with high slenderness ratios $s \geq 300$ with low stiffness and relatively low buckling loads.

In this paper, active buckling control of a relatively stiff beam-column with circular cross-section and relatively low slenderness ratio $s=108$ that is closer to the limiting slenderness ratio of $s_{\lim }=38$ of the chosen material is investigated experimentally. A new concept for piezo-elastic supports is used in which lateral forces of piezoelectric stack actuators are transformed into bending moments acting in arbitrary directions at the beam-column ends. By that, the beam-column surface is kept entirely free of any actuators. In [11], active buckling control for this beam-column system was simulated numerically. A lateral impulse disturbance force was used to initially deform the ideal beam-column and an increase of $247 \%$ in the critical buckling load was achieved by LQR control. In a real test setup this proved to be insufficient and, therefore, an integral LQR will now be used to compensate the deformation of the beam-column due to increasing axial load.

\section{System description and mathematical model}

The investigated system is a slender beam-column made of aluminum alloy EN AW7075 with length $l_{\mathrm{b}}$. It has a circular solid cross-section of radius $r_{\mathrm{b}}$, bending stiffness $E I_{\mathrm{b}}$ and density $\varrho_{\mathrm{b}}$, all assumed to be constant across the entire beam-column length, figure 1. The beam-column has two piezo-elastic supports $\mathrm{A}$ at $x=0$ and $\mathrm{B}$ at $x=l_{\mathrm{b}}$. At the upper support B, a constant axial load $F_{x}$ is applied. Strain gauges at positions $x_{\mathrm{s}, 1 / 2}$ are used to measure the surface strain of the beam-column in $y$ - and $z$-direction. 


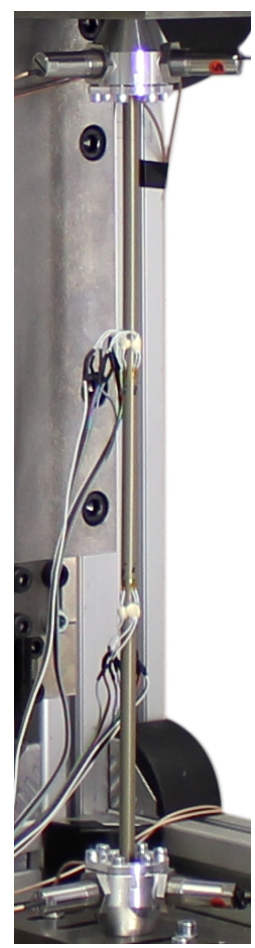

(a)

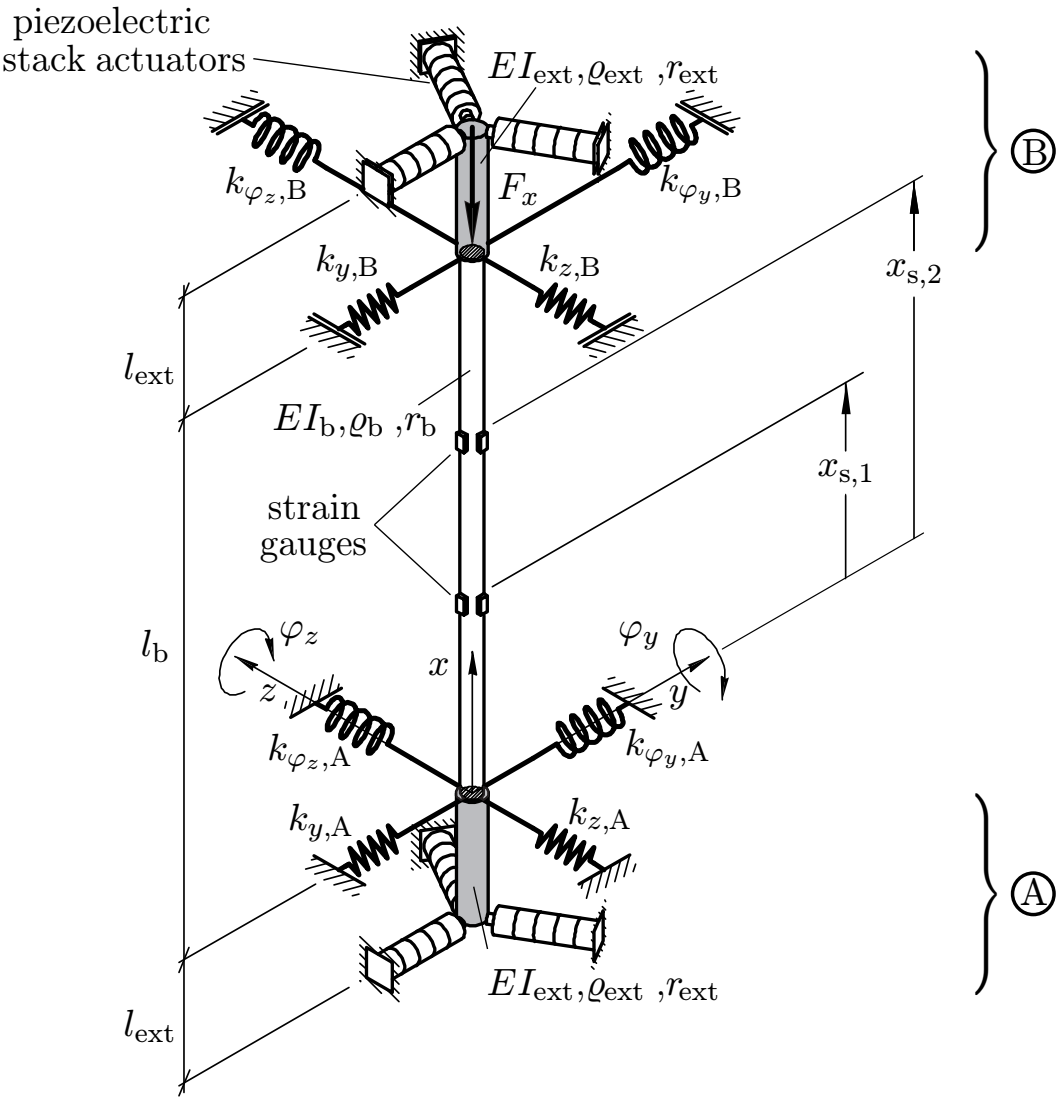

(b)

Figure 1: Beam-column system, (a) beam-column with piezo-elastic supports for experimental test, (b) sketch of beam-column.

Figure 2 shows a sectional view of piezo-elastic support A. The central element of the support is an elastic spring element made of spring steel 1.4310 that bears the axial compressive load and allows rotations in any plane perpendicular to the $x$-axis. It is represented by rotational stiffness $k_{\varphi_{y}, \mathrm{~A}}=k_{\varphi_{z}, \mathrm{~A}}=k_{\varphi_{y}, \mathrm{~B}}=k_{\varphi_{z}, \mathrm{~B}}=k_{\mathrm{r}}$ and lateral stiffness $k_{y, \mathrm{~A}}=k_{z, \mathrm{~A}}=k_{y, \mathrm{~B}}=k_{z, \mathrm{~B}}=k_{\mathrm{l}}$ that are the same for both supports $\mathrm{A}$ and $\mathrm{B}$ and in both $y$ - and $z$-direction, figure $1 \mathrm{~b}$.

In each piezo-elastic support $\mathrm{A}$ and $\mathrm{B}$ at $x=-l_{\text {ext }}$ and $x=l_{\mathrm{b}}+l_{\text {ext }}$, three piezoelectric stack actuators are arranged in the support housing made of aluminum alloy EN AW-7075 at an angle of $120^{\circ}$ to each other in one plane orthogonal to the beam-column's $x$-axis. They are connected to the beam-column via a relatively stiff axial extension made of hardened steel 1.2312 with length $l_{\text {ext }}$, radius $r_{\text {ext }}$, bending stiffness $E I_{\text {ext }}$ and density $\varrho_{\text {ext }}$ creating cantilever beam ends beyond both elastic spring elements. This way, active lateral forces in arbitrary directions orthogonal to the beamcolumn's longitudinal $x$-axis are transformed into bending moments acting in arbitrary directions at the beam-column ends in both piezo-elastic supports A and B. 


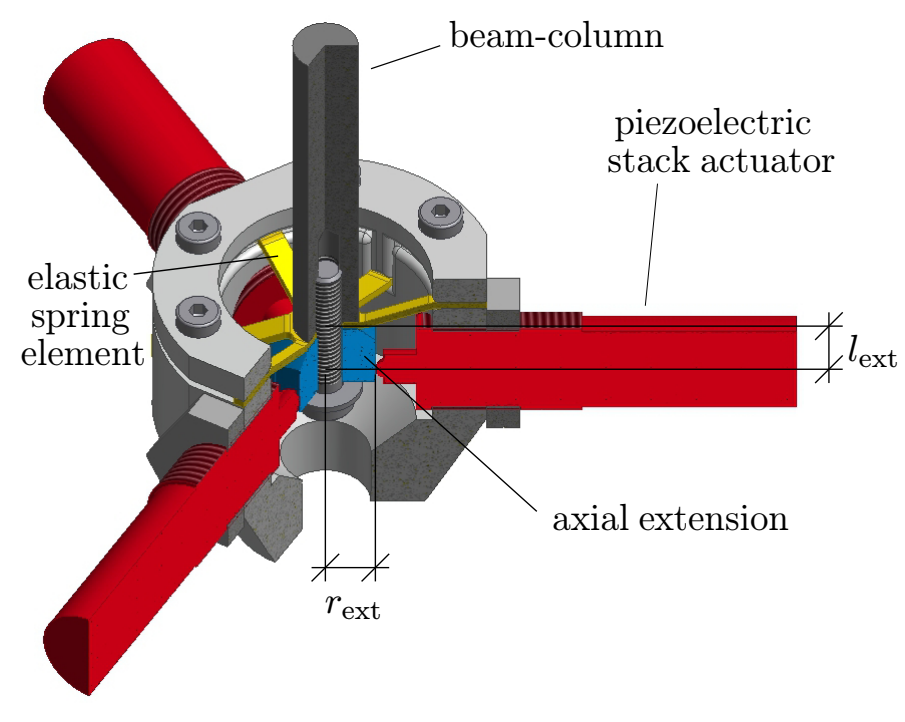

Figure 2: Sectional view of piezo-elastic support A.

The parameters of the beam-column system presented in figures 1 and 2 are summarized in table 1 . The values for the spring element's rotational stiffness $k_{\mathrm{r}}$ and lateral stiffness $k_{1}$ were obtained from static stiffness measurements on a material testing machine, [12].

Table 1: Properties of active beam-column system.

\begin{tabular}{llcl}
\hline property & symbol & value & SI-unit \\
\hline beam-column length & $l_{\mathrm{b}}$ & $400.0 \cdot 10^{-3}$ & $\mathrm{~m}$ \\
beam-column radius & $r_{\mathrm{b}}$ & $4.0 \cdot 10^{-3}$ & $\mathrm{~m}$ \\
beam-column density & $\varrho_{\mathrm{b}}$ & 2789.0 & $\mathrm{~kg} / \mathrm{m}^{3}$ \\
beam-column Young's modulus & $E_{\mathrm{b}}$ & $75.8 \cdot 10^{9}$ & $\mathrm{~N} / \mathrm{m}^{2}$ \\
beam-column proportional limit & $\sigma_{\mathrm{p}, \mathrm{b}}$ & $485.0 \cdot 10^{6}$ & $\mathrm{~N} / \mathrm{m}^{2}$ \\
axial extension length & $l_{\text {ext }}$ & $7.5 \cdot 10^{-3}$ & $\mathrm{~m}$ \\
axial extension radius & $r_{\text {ext }}$ & $6.0 \cdot 10^{-3}$ & $\mathrm{~m}$ \\
axial extension density & $\varrho_{\text {ext }}$ & 7810.0 & $\mathrm{~kg} / \mathrm{m}^{3}$ \\
axial extension Young's modulus & $E_{\text {ext }}$ & $210.0 \cdot 10^{9}$ & $\mathrm{~N} / \mathrm{m}^{2}$ \\
spring element rotational stiffness & $k_{\mathrm{r}}$ & 192.4 & $\mathrm{Nm} / \mathrm{rad}$ \\
spring element lateral stiffness & $k_{\mathrm{l}}$ & $30.0 \cdot 10^{6}$ & $\mathrm{~N} / \mathrm{m}^{2}$ \\
piezoelectric stack actuator lateral stiffness & $k_{\mathrm{p}}$ & $22.0 \cdot 10^{6}$ & $\mathrm{~N} / \mathrm{m}$ \\
strain gauge position 1 & $x_{\mathrm{s}, 1}$ & $148.0 \cdot 10^{-3}$ & $\mathrm{~m}$ \\
strain gauge position 2 & $x_{\mathrm{s}, 2}$ & $252.0 \cdot 10^{-3}$ & $\mathrm{~m}$ \\
modal damping ratio mode 1 & $\zeta_{1}$ & $15.0 \cdot 10^{-3}$ & - \\
modal damping ratio mode 2 & $\zeta_{2}$ & $4.5 \cdot 10^{-3}$ & - \\
\hline
\end{tabular}

For experimental investigations, the beam-column system is installed in an experimental test setup. Figure 3 shows a photo and a CAD sketch of the experimental test setup for the active buckling control of the slender beam-column with circular 
cross-section and piezo-elastic supports, 1, figure 1a. Piezo-elastic support A is fixed on a baseplate, 2, and piezo-elastic support B is fixed to a parallel guidance, 3, that is connected to a stiff frame structure, 4. The beam-column is loaded via a high lever ratio by a mass of $25 \mathrm{~kg}, 5$, that is placed on a hinged beam, 6 . The lever ratio and, therefore, the axial load are increased by shifting the mass in nearly horizontal plane via a linear axle, 7 , operated by a stepper motor, 8 . With that, the axial loads range between the static load $F_{\text {stat }}=260 \mathrm{~N}$ of the parallel guidance, 3 , and $F_{\max } \approx 4000 \mathrm{~N}$. The axial load is measured by a load cell, 9 .

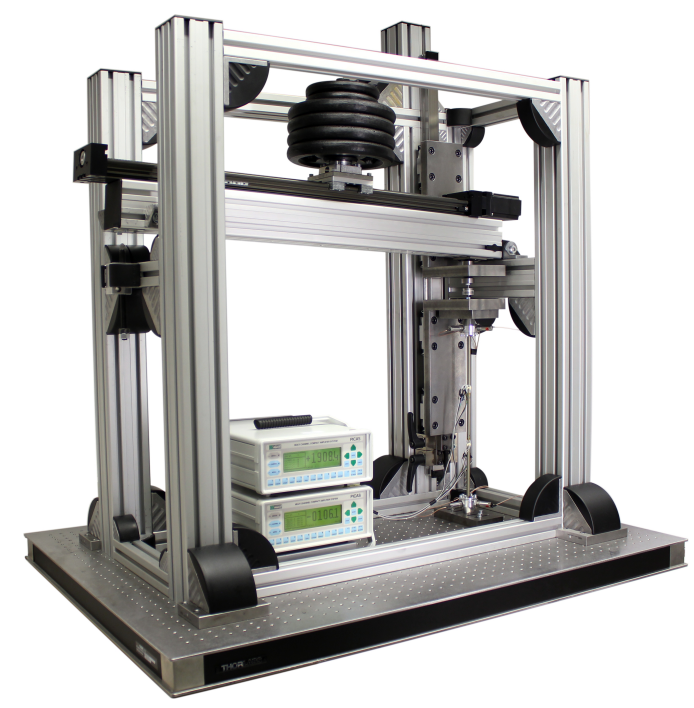

(a)

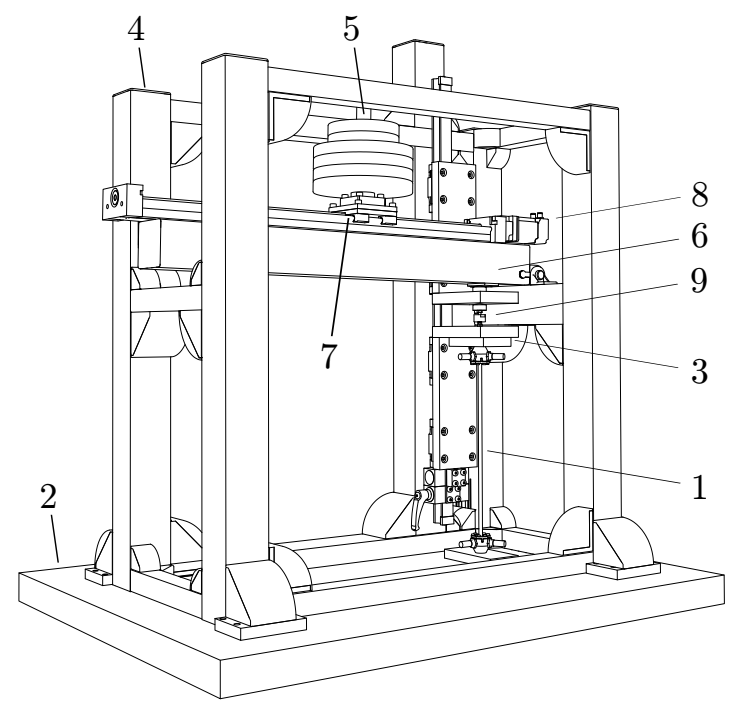

(b)

Figure 3: Experimental test setup for active buckling control, (a) photo of test setup, (b) CAD sketch of test setup (without strain gauge amplifiers).

\subsection{Finite Element model}

The beam-column and the stiff axial extensions are discretized by $N-1$ one-dimensional Euler-Bernoulli beam elements of length $l_{\mathrm{el}}$ with $N$ nodes. Each node $n$ is described by the lateral displacements $v_{n}$ and $w_{n}$ in $y$ - and $z$-direction and the rotational displacements $\varphi_{y, n}$ and $\varphi_{z, n}$ around the $y$ - and $z$-axis, figure 4a. Axial and rotational displacements in and around the $x$-axis of the beam-column are neglected. Figure $4 \mathrm{~b}$ shows the discretized beam-column with $N-1$ finite elements and $N$ nodes. The axial extensions are discretized by one single finite element, so that the constant axial load $F_{x}$ acts at node $n=N-1$. The piezoelectric stack actuators in the piezo-elastic supports $\mathrm{A}$ and $\mathrm{B}$ are represented by lateral stiffness $k_{\mathrm{p}}$ and additional active control forces $F_{\mathrm{a} y / z, \mathrm{~A} / \mathrm{B}}(t)$ in $y$ - and $z$-direction of nodes $n=1$ and $n=N$ of the FE model. 


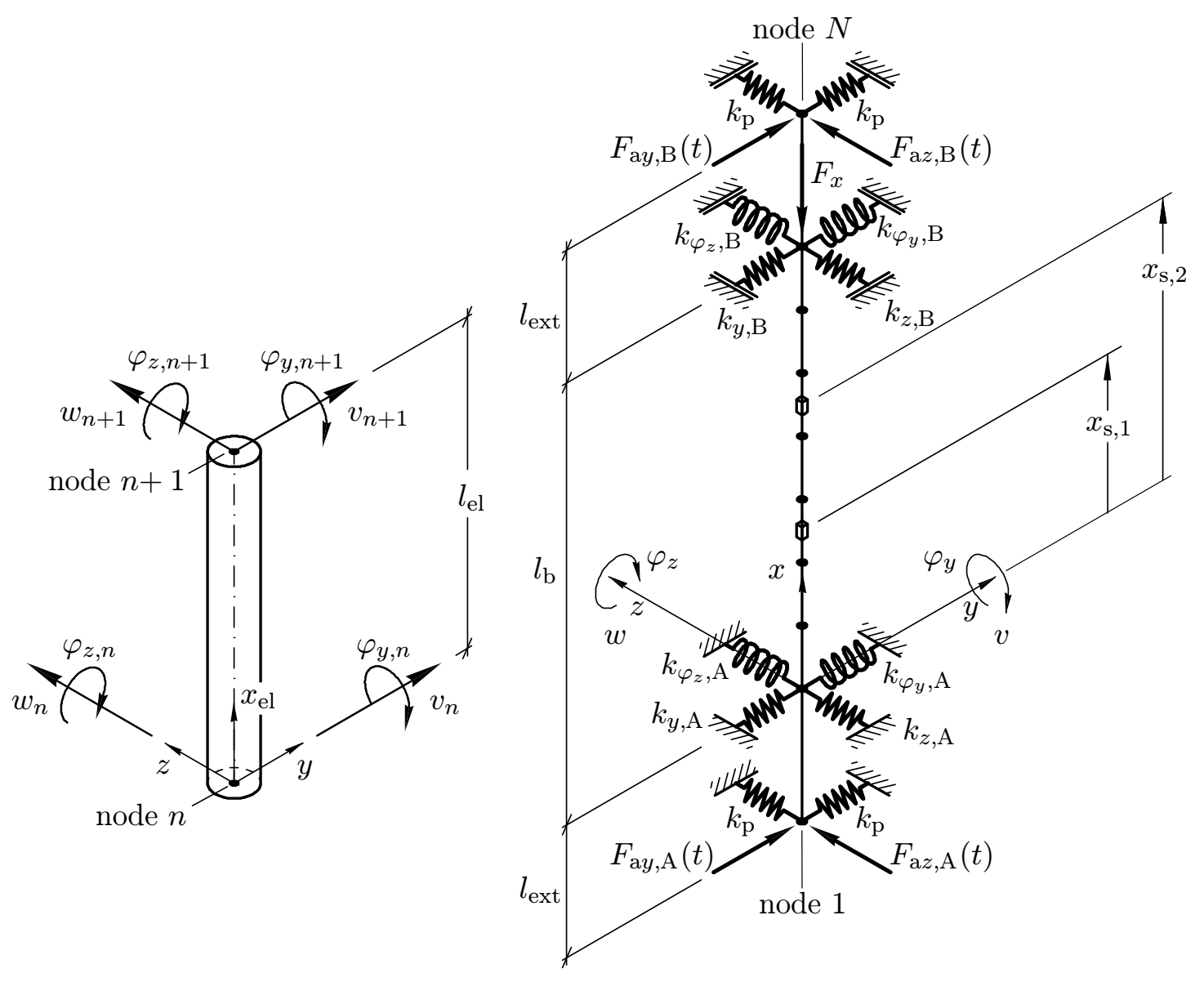

(a)

(b)

Figure 4: FE beam-column system, (a) $n$-th finite beam-column element of length $l_{\mathrm{el}}$ with element coordinates in positive directions, (b) FE model of beam-column.

The Galerkin method with cubic Hermitian shape functions $\boldsymbol{g}\left(x_{\mathrm{el}}\right)$ is used to get the element mass matrix $\boldsymbol{M}_{\mathrm{el}}$, elastic element stiffness matrix $\boldsymbol{K}_{\mathrm{e}, \mathrm{el}}$ and geometric element stiffness matrix $\boldsymbol{K}_{\mathrm{g}, \mathrm{el}}$, all $[8 \times 8$ ], for the EulER-BERnOulli beam elements, [13]. The element mass matrix $\boldsymbol{M}_{\mathrm{el}}$ and elastic element stiffness matrix $\boldsymbol{K}_{\mathrm{e}, \mathrm{el}}$ are readily found in literature, $[13,14]$. The geometric element stiffness matrix

$$
\boldsymbol{K}_{\mathrm{g}, \mathrm{el}}=\frac{1}{l_{\mathrm{el}}}\left(\begin{array}{cccccccc}
6 / 5 & 0 & 0 & l_{\mathrm{el}} / 10 & -6 / 5 & 0 & 0 & l_{\mathrm{el}} / 10 \\
& 6 / 5 & -l_{\mathrm{el}} / 10 & 0 & 0 & -6 / 5 & -l_{\mathrm{el}} / 10 & 0 \\
& & 2 / 15 l_{\mathrm{el}}^{2} & 0 & 0 & l_{\mathrm{el}} / 10 & -l_{\mathrm{el}}^{2} / 30 & 0 \\
& & & 2 / 15 l_{\mathrm{el}}^{2} & -l_{\mathrm{el}} / 10 & 0 & 0 & -l_{\mathrm{el}}^{2} / 30 \\
& & & & 6 / 5 & 0 & 0 & -l_{\mathrm{el}} / 10 \\
& & & & & 6 / 5 & l_{\mathrm{el}} / 10 & 0 \\
& & & & & & 2 / 15 l_{\mathrm{el}}^{2} & 0 \\
& & & & & & & 2 / 15 l_{\mathrm{el}}^{2}
\end{array}\right)
$$

describes the influence of axial load $F_{x}$ on the beam-column's lateral stiffness. After assembling the global mass and stiffness matrices from the element matrices, the FE 
equation of motion is

$$
\boldsymbol{M} \ddot{\boldsymbol{r}}(t)+\boldsymbol{D} \dot{\boldsymbol{r}}(t)+\left(\boldsymbol{K}_{\mathrm{e}}-F_{x} \boldsymbol{K}_{\mathrm{g}}\right) \boldsymbol{r}(t)=\boldsymbol{B}_{0} \boldsymbol{u}(t)
$$

with the $[4 N \times 1] \mathrm{FE}$ displacement vector

$$
\boldsymbol{r}(t)=\left[v_{1}(t), w_{1}(t), \varphi_{y, 1}(t), \varphi_{z}(t), \ldots, v_{N}(t), w_{N}(t), \varphi_{y, N}(t), \varphi_{z, N}(t)\right]^{T} .
$$

In (2), $\boldsymbol{M}$ is the global mass matrix, $\boldsymbol{D}$ is the global damping matrix, $\boldsymbol{K}_{\mathrm{e}}$ is the global elastic stiffness matrix and $\boldsymbol{K}_{\mathrm{g}}$ is the global geometric stiffness matrix, all $[4 N \times 4 N] . \quad \boldsymbol{K}_{\mathrm{e}}$ also includes the discrete stiffness $k_{\mathrm{p}}, k_{\mathrm{l}}$ and $k_{\mathrm{r}}$ of piezoelectric stack actuators and elastic spring elements, table 1 . The lateral stiffness $k_{\mathrm{p}}$ is added to the entries of the lateral degrees of freedom of nodes $n=1$ and $n=N$. Similarly, the lateral and rotational stiffness $k_{1}$ and $k_{\mathrm{r}}$ are added to the entries of the lateral and rotational degrees of freedom of nodes $n=2$ and $n=N-1$, figure 4 b. $\boldsymbol{D}$ is assumed by RAYLEIGH proportional damping $\boldsymbol{D}=\alpha \boldsymbol{M}+\beta \boldsymbol{K}_{\mathrm{e}},[15]$. The proportional damping coefficients $\alpha$ and $\beta$ are determined for experimentally identified modal damping ratios $\zeta_{1 / 2}$ of the first two bending modes, figure 5 a.

The right side of (2) represents the active control forces that are summarized in control input vector

$$
\boldsymbol{u}(t)=\left[\begin{array}{c}
F_{\mathrm{a} y, \mathrm{~A}}(t) \\
F_{\mathrm{a} z, \mathrm{~A}}(t) \\
F_{\mathrm{a} y, \mathrm{~B}}(t) \\
F_{\mathrm{a} z, \mathrm{~B}}(t)
\end{array}\right] .
$$

The $[4 N \times 4]$ control input matrix $\boldsymbol{B}_{0}$ allocates the active forces to the lateral degrees of freedom of the first and last nodes $n=1$ and $n=N$ of the FE model.

The beam-column's lateral displacements $v(t)$ and $w(t)$ in the real test setup are not measured directly. Four strain gauges at each of the two strain gauge positions $x_{\mathrm{s}, 1 / 2}$ measure the surface strains due to bending in $y$ - and $z$-direction, figure 1 . The surface strains in $y$ - and $z$-direction at the strain gauge positions are chosen as output

$$
\boldsymbol{y}(t)=\left[\begin{array}{c}
\varepsilon_{y}\left(x_{\mathrm{s}, 1}, t\right) \\
\varepsilon_{z}\left(x_{\mathrm{s}, 1}, t\right) \\
\varepsilon_{y}\left(x_{\mathrm{s}, 2}, t\right) \\
\varepsilon_{z}\left(x_{\mathrm{s}, 2}, t\right)
\end{array}\right]=\boldsymbol{C}_{0} \boldsymbol{r}(t)
$$

of the FE model. In (5), the $[4 \times 4 N]$ output matrix $\boldsymbol{C}_{0}$ allocates the surface strains $\boldsymbol{y}(t)$ to the FE displacement vector (3) by the entries $-r_{\mathrm{b}} \boldsymbol{g}^{\prime \prime}\left(x_{\mathrm{s}, 1 / 2}\right)$ at the FE nodes surrounding strain gauge positions $x_{\mathrm{s}, 1 / 2} \cdot r_{\mathrm{b}}$ is the beam-column radius and $\boldsymbol{g}^{\prime \prime}\left(x_{\mathrm{s}, 1 / 2}\right)$ the second derivative of the HERMITIAN shape functions at the strain gauge positions, [13].

For convenience, (2) and (5) are written in state space representation

$$
\begin{aligned}
& \dot{\boldsymbol{x}}_{\mathrm{FE}}(t)=\underbrace{\left(\left[\begin{array}{cc}
\mathbf{0} & \mathbf{I} \\
-\boldsymbol{M}^{-1} \boldsymbol{K}_{\mathrm{e}} & -\boldsymbol{D}
\end{array}\right]+F_{x}\left[\begin{array}{cc}
\mathbf{0} & \mathbf{0} \\
\boldsymbol{M}^{-1} \boldsymbol{K}_{\mathrm{g}} & \mathbf{0}
\end{array}\right]\right)}_{[8 N \times 8 N]} \boldsymbol{x}_{\mathrm{FE}}(t)+\underbrace{\left[\begin{array}{c}
\mathbf{0} \\
\boldsymbol{M}^{-1} \boldsymbol{B}_{0}
\end{array}\right]}_{[8 N \times 4]} \boldsymbol{u}(t) \\
& \boldsymbol{y}(t)=\underbrace{\left[\begin{array}{ll}
\boldsymbol{C}_{0} & \mathbf{0}
\end{array}\right]}_{[4 \times 8 N]} \boldsymbol{x}_{\mathrm{FE}}(t),
\end{aligned}
$$


with $[8 N \times 1]$ FE state vector $\boldsymbol{x}_{\mathrm{FE}}(t)=[\boldsymbol{r}(t), \dot{\boldsymbol{r}}(t)]^{T}$ and zero and identity matrices $\mathbf{0}$ and $\mathbf{I}$ of appropriate dimensions. In short form, (6) can be written as

$$
\begin{aligned}
& \dot{\boldsymbol{x}}_{\mathrm{FE}}(t)=\boldsymbol{A}_{\mathrm{FE}}\left(F_{x}\right) \boldsymbol{x}_{\mathrm{FE}}(t)+\boldsymbol{B}_{\mathrm{FE}} \boldsymbol{u}(t) \\
& \boldsymbol{y}(t)=\boldsymbol{C}_{\mathrm{FE}} \boldsymbol{x}_{\mathrm{FE}}(t)
\end{aligned}
$$

representing the full FE state space model of the beam-column. The influence of axial load $F_{x}$ on the system behavior is described by the system matrix $\boldsymbol{A}_{\mathrm{FE}}\left(F_{x}\right)$.

\subsection{System identification and model validation}

To validate the FE beam-column model, the transfer behavior of model (7) and experiment are compared. In a first step, (7) is transformed into LAPLACE domain

$$
\begin{array}{ll}
s \boldsymbol{x}_{\mathrm{FE}}(s) & =\boldsymbol{A}_{\mathrm{FE}}\left(F_{x}\right) \boldsymbol{x}_{\mathrm{FE}}(s)+\boldsymbol{B}_{\mathrm{FE}} \boldsymbol{u}(s) \\
\boldsymbol{y}(s) & =\boldsymbol{C}_{\mathrm{FE}} \boldsymbol{x}_{\mathrm{FE}}(s) .
\end{array}
$$

Replacing the state vector $\boldsymbol{x}_{\mathrm{FE}}(s)$ in $(8)$, the $[4 \times 4]$ matrix of frequency response functions as the relation of the active forces in control input vector (4) to the strain gauge outputs (5) is

$$
\boldsymbol{G}_{\mathrm{FE}}\left(F_{x}, s\right)=\frac{\boldsymbol{y}(s)}{\boldsymbol{u}(s)}=\boldsymbol{C}_{\mathrm{FE}}\left[s \mathbf{I}-\boldsymbol{A}_{\mathrm{FE}}\left(F_{x}\right)\right]^{-1} \boldsymbol{B}_{\mathrm{FE}}
$$

[16]. The two frequency response functions

$$
G_{y}\left(F_{x}, s\right)=\frac{\varepsilon_{y}\left(x_{\mathrm{s}, 1}, s\right)}{F_{\mathrm{a} y, \mathrm{~A}}(s)} \quad \text { and } \quad G_{z}\left(F_{x}, s\right)=\frac{\varepsilon_{z}\left(x_{\mathrm{s}, 1}, s\right)}{F_{\mathrm{a} z, \mathrm{~A}}(s)}
$$

represent the strain responses $\varepsilon_{y / z}$ at the lower strain gauge position $x_{\mathrm{s}, 1}$ to the excitation by active forces $F_{\mathrm{a} y / z, \mathrm{~A}}$ in piezo-elastic support A.

Figure 5a shows amplitude $\left|G_{y / z}\right|$ and phase $\arg G_{y / z}$ of the frequency response functions $G_{y}$ and $G_{z}$ for the beam-column loaded with axial load $F_{x}=2000 \mathrm{~N}$ for model and experiment. Both are plotted as functions of frequency $f$ in $\mathrm{Hz}$ using the conversion $s=j 2 \pi f$. The lateral and rotational stiffness $k_{1}$ and $k_{\mathrm{r}}$ of the elastic spring element as well as the modal damping ratios in table 1 were manually tuned to fit the resonance frequencies and amplitudes. The frequency response functions from model and experiment coincide well for the first three resonance frequencies in the considered frequency range 0 to $1200 \mathrm{~Hz}$.

The FE model (7) also shows the beam-column's dependency of axial load $F_{x}$, figure 5b. In the numerical simulation, the eigenfrequencies $f_{i}\left(F_{x}\right)$ are calculated by solution of the eigenvalue problem

$$
\operatorname{det}\left[\left(\boldsymbol{K}_{\mathrm{e}}-F_{x} \boldsymbol{K}_{\mathrm{g}}\right)-(2 \pi f)^{2} \boldsymbol{M}\right]=0 .
$$

The eigenfrequencies decrease with increasing axial load and reach zero for the buckling loads that are calculated from the global elastic and geometric stiffness matrices by solution of the eigenvalue problem

$$
\operatorname{det}\left[\boldsymbol{K}_{\mathrm{e}}-F_{x} \boldsymbol{K}_{\mathrm{g}}\right]=0,
$$



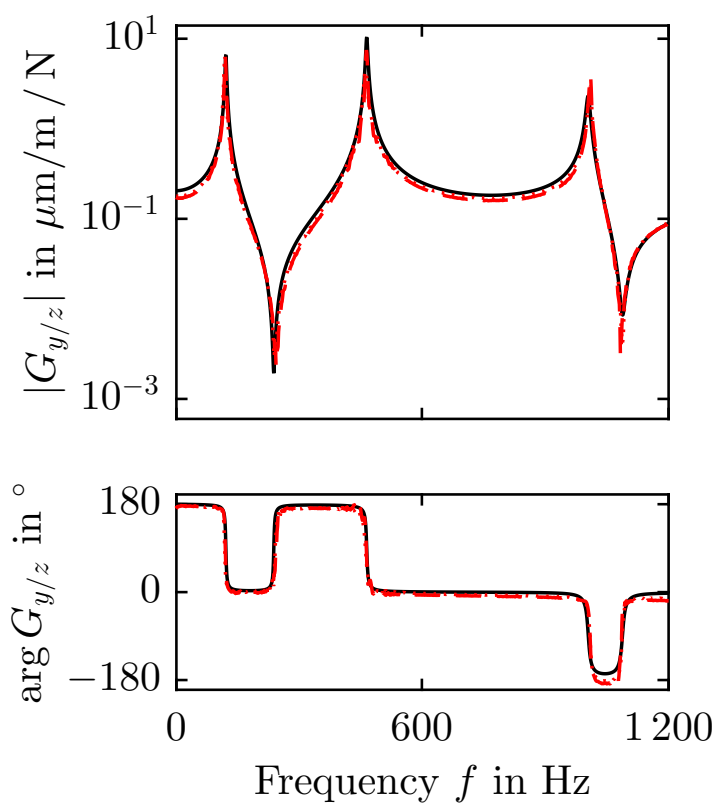

(a)

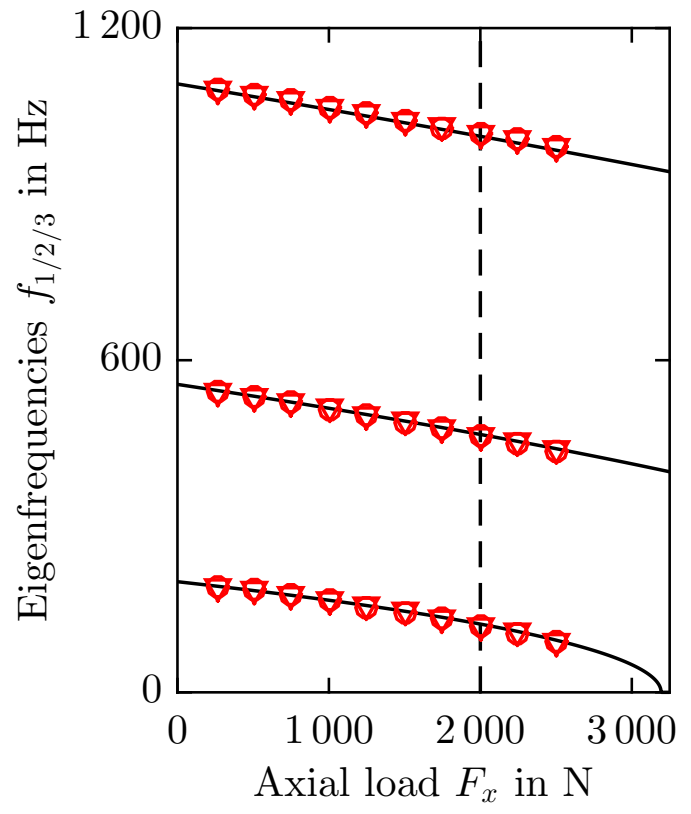

(b)

Figure 5: System identification of beam-column system, (a) amplitude $\left|G_{y / z}\right|$ and phase $\arg G_{y / z}$ of frequency response functions $G_{y / z}$ for axial load $F_{x}=2000 \mathrm{~N}$ from numerical simulation $(-)$ and experiments in $y$-direction $(---)$ and $z$-direction $(\cdots \ldots)$, (b) first three eigenfrequencies $f_{1 / 2 / 3}$ for varying axial loads $F_{x}$ from simulation $(-)$ and experiments in $y$-direction $(\nabla)$ and $z$-direction $(O)$.

[14]. The lowest buckling load is the critical buckling load $F_{x, \text { cr }}$ at which the first eigenfrequency $f_{1}$ reaches zero. It is determined for the given boundary conditions to $F_{x, c r}=3194 \mathrm{~N}$. In the experiment, the system identification can only be performed up to axial loads of $F_{x}=2500 \mathrm{~N}$ due to the high static deformations of the uncontrolled beam-column, figure 7 .

\section{Controller design}

In the following, first a reduced modal model of the axially loaded beam-column is presented. Second, the modal model is augmented to include integrated states and then a LQR for the active buckling control is derived.

\subsection{Reduced modal model}

For the full state FE model (2), a number of $N=35$ nodes resulting in $4 N=140$ degrees of freedom is chosen to properly describe the maximum surface strains at the strain gauge positions $x_{\mathrm{s}, 1 / 2}$ according to (5). For controller design, however, the FE model is reduced by modal truncation to only include the first two modes for both $y$ - and $z$-direction. Consequently, the FE displacement vector (3) is approximated by modal 
displacements

$$
\boldsymbol{q}_{\mathrm{m}}(t)=\left[\begin{array}{c}
q_{1, y}(t) \\
q_{1, z}(t) \\
q_{2, y}(t) \\
q_{2, z}(t)
\end{array}\right]
$$

via the transformation

$$
\boldsymbol{r}(t) \approx \boldsymbol{\Phi} \boldsymbol{q}_{\mathrm{m}}(t) .
$$

The $[4 N \times 4]$ modal matrix

$$
\boldsymbol{\Phi}=\left[\widehat{\boldsymbol{r}}_{1, y}, \widehat{\boldsymbol{r}}_{1, z}, \widehat{\boldsymbol{r}}_{2, y}, \widehat{\boldsymbol{r}}_{2, z}\right]
$$

includes the first two $[4 N \times 1]$ eigenvectors $\widehat{\boldsymbol{r}}_{1}$ and $\widehat{\boldsymbol{r}}_{2}$ in $y$ - and $z$-direction of the FE Model, [17]. The eigenvectors in (15) are normalized with respect to mass matrix $\boldsymbol{M}$ leading to the modal mass matrix $\widetilde{\boldsymbol{M}}$, modal elastic stiffness matrix $\widetilde{\boldsymbol{K}}_{\mathrm{e}}$, modal geometric stiffness matrix $\widetilde{\boldsymbol{K}}_{\mathrm{g}}$ and modal damping matrix $\widetilde{\boldsymbol{D}}$

$$
\begin{aligned}
\widetilde{\boldsymbol{M}} & =\boldsymbol{\Phi}^{T} \boldsymbol{M} \boldsymbol{\Phi}=\mathbf{I} \\
\widetilde{\boldsymbol{K}}_{\mathrm{e}} & =\boldsymbol{\Phi}^{T} \boldsymbol{K}_{\mathrm{e}} \boldsymbol{\Phi} \\
\widetilde{\boldsymbol{K}}_{\mathrm{g}} & =\boldsymbol{\Phi}^{T} \boldsymbol{K}_{\mathrm{g}} \boldsymbol{\Phi} \\
\widetilde{\boldsymbol{D}} & =\boldsymbol{\Phi}^{T} \boldsymbol{D} \boldsymbol{\Phi}
\end{aligned}
$$

with identity matrix $\mathbf{I}$, all $[4 \times 4]$. Using the $[8 \times 1]$ modal state vector with the modal displacements and velocities

$$
\boldsymbol{x}_{\mathrm{m}}(t)=\left[\begin{array}{c}
\boldsymbol{q}_{\mathrm{m}}(t) \\
\dot{\boldsymbol{q}}_{\mathrm{m}}(t)
\end{array}\right],
$$

the modal state space equations of first order are

$$
\begin{aligned}
& \dot{\boldsymbol{x}}_{\mathrm{m}}(t)=\underbrace{\left(\left[\begin{array}{cc}
\mathbf{0} & \mathbf{I} \\
-\widetilde{\boldsymbol{K}}_{\mathrm{e}} & -\widetilde{\boldsymbol{D}}
\end{array}\right]+F_{x}\left[\begin{array}{cc}
\mathbf{0} & \mathbf{0} \\
\widetilde{\boldsymbol{K}}_{\mathrm{g}} & \mathbf{0}
\end{array}\right]\right)}_{[8 \times 8]} \boldsymbol{x}_{\mathrm{m}}(t)+\underbrace{\left[\begin{array}{c}
\mathbf{0} \\
\boldsymbol{\Phi}^{T} \boldsymbol{B}_{0}
\end{array}\right]}_{[8 \times 4]} \boldsymbol{u}(t) \\
& \boldsymbol{y}(t)=\underbrace{\left[\begin{array}{ll}
\boldsymbol{C}_{0} \boldsymbol{\Phi} & \mathbf{0}
\end{array}\right]}_{[4 \times 8]} \boldsymbol{x}_{\mathrm{m}}(t),
\end{aligned}
$$

[17]. (18) can also be written in short form

$$
\begin{aligned}
& \dot{\boldsymbol{x}}_{\mathrm{m}}(t)=\boldsymbol{A}_{\mathrm{m}}\left(F_{x}\right) \boldsymbol{x}_{\mathrm{m}}(t)+\boldsymbol{B}_{\mathrm{m}} \boldsymbol{u}(t) \\
& \boldsymbol{y}(t)=\boldsymbol{C}_{\mathrm{m}} \boldsymbol{x}_{\mathrm{m}}(t) .
\end{aligned}
$$

Similar to the transfer behavior of the FE model (9), the matrix of frequency response functions of the reduced modal model is obtained by

$$
\boldsymbol{G}_{\mathrm{m}}\left(F_{x}, s\right)=\frac{\boldsymbol{y}\left(F_{x}, s\right)}{\boldsymbol{u}(s)}=\boldsymbol{C}_{\mathrm{m}}\left[s \mathbf{I}-\boldsymbol{A}_{\mathrm{m}}\left(F_{x}\right)\right]^{-1} \boldsymbol{B}_{\mathrm{m}} .
$$

Figure 6 shows the comparison of amplitude $\left|G_{y / z}\right|$ and phase $\arg G_{y / z}$ of the frequency response functions (10) for the full FE model (7) and the reduced modal model (19) for the beam-column loaded with axial load $F_{x}=2000 \mathrm{~N}$. 

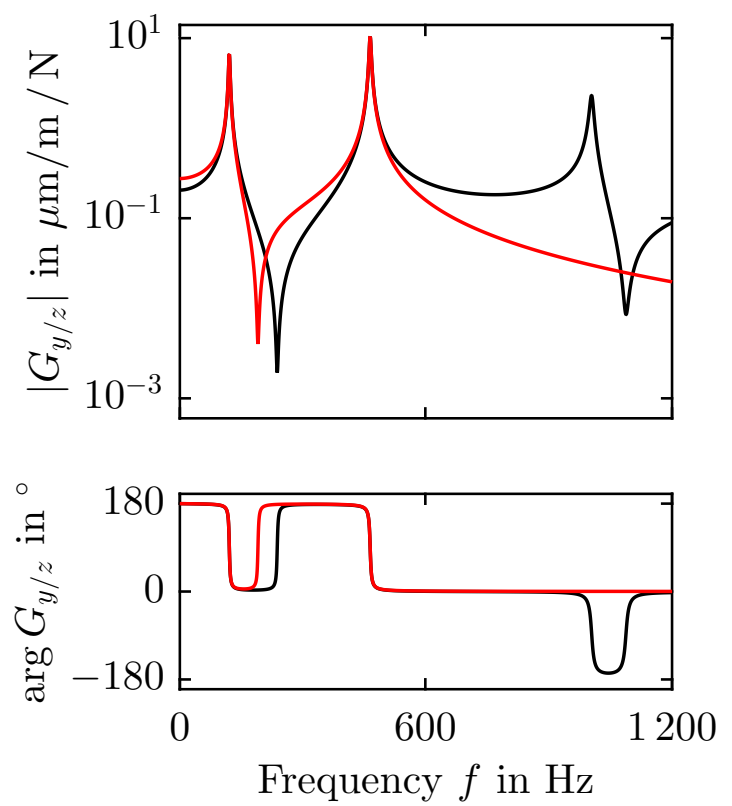

Figure 6: Amplitude $\left|G_{y / z}\right|$ and phase $\arg G_{y / z}$ of the frequency transfer functions $G_{y / z}$ for axial load $F_{x}=2000 \mathrm{~N}$ from full FE model ( - ) and reduced modal model ( - ).

The reduced modal model only captures the system dynamics for the first two resonance frequencies at $f_{1}=123 \mathrm{~Hz}$ and $f_{2}=466 \mathrm{~Hz}$. The resonance frequencies coincide with the full FE model (7) whereas the zero between first and second resonance as well as the static amplification differ which is attributed to the influence of higher modes in the low frequency range that are neglected in the reduced modal model, [16, 17]. The strain gauge positions $x_{\mathrm{s}, 1 / 2}$, table 1 , are chosen such that the deviations of full and reduced model are minimal so that the possible control and observation spillover is reduced.

\subsection{Integral $L Q R$}

The chosen type of controller for active buckling control is a linear quadratic regulator (LQR). Due to imperfections such as predeformation, eccentric loading or clamping moments that are present in a real beam-column system, the controller needs to have an additional integral term to avoid a static controller error. Therefore, the modal state vector (17) is augmented by the integral of the modal displacements

$$
\boldsymbol{x}_{\mathrm{i}}(t)=\int \boldsymbol{q}_{\mathrm{m}}(t) \mathrm{d} t
$$

to get the new $[12 \times 1]$ state vector

$$
\boldsymbol{x}(t)=\left[\begin{array}{c}
\boldsymbol{x}_{\mathrm{m}}(t) \\
\boldsymbol{x}_{\mathrm{i}}(t)
\end{array}\right],
$$


$[18,19]$. With the first derivative of the new state vector

$$
\dot{\boldsymbol{x}}_{\mathrm{i}}(t)=\boldsymbol{q}_{\mathrm{m}}(t)=\left[\begin{array}{ll}
\mathbf{I} & \mathbf{0}
\end{array}\right] \boldsymbol{x}_{\mathrm{m}}(t),
$$

the augmented state space system including the integral term can be written as

$$
\begin{aligned}
& \dot{\boldsymbol{x}}(t)=\underbrace{\left[\begin{array}{cc}
\boldsymbol{A}_{\mathrm{m}}\left(F_{x}\right) & \mathbf{0} \\
{\left[\begin{array}{ll}
\mathbf{I} & \mathbf{0}
\end{array}\right]} & \mathbf{0}
\end{array}\right]}_{[12 \times 12]} \boldsymbol{x}(t)+\underbrace{\left[\begin{array}{c}
\boldsymbol{B}_{\mathrm{m}} \\
\mathbf{0}
\end{array}\right]}_{[12 \times 4]} \boldsymbol{u}(t) \\
& \boldsymbol{y}(t)=\underbrace{\left[\begin{array}{ll}
\boldsymbol{C}_{\mathrm{m}} & \mathbf{0}
\end{array}\right]}_{[4 \times 12]} \boldsymbol{x}(t) .
\end{aligned}
$$

In short form, the final state space system (24) of the beam-column system in figure $4 \mathrm{~b}$ is written as

$$
\begin{aligned}
& \dot{\boldsymbol{x}}(t)=\boldsymbol{A}\left(F_{x}\right) \boldsymbol{x}(t)+\boldsymbol{B} \boldsymbol{u}(t) \\
& \boldsymbol{y}(t)=\boldsymbol{C} \boldsymbol{x}(t) .
\end{aligned}
$$

Active buckling control of the circular beam-column is achieved by an infinite horizon, continuous-time linear quadratic regulator (LQR). The control law determines the control input $\boldsymbol{u}(t)$ so that the quadratic performance index

$$
J=\int_{0}^{\infty}\left\{\boldsymbol{x}^{T}(t) \boldsymbol{Q} \boldsymbol{x}(t)+\boldsymbol{u}^{T}(t) \boldsymbol{R} \boldsymbol{u}(t)\right\} \mathrm{d} t
$$

is minimized, [19]. The $[12 \times 12]$ matrix $\boldsymbol{Q}$ includes weights on system state vector $\boldsymbol{x}(t)$ and is chosen as diagonal matrix with largest weights on the modal displacements of the first modes in $y$ - and $z$-direction and their integrals, [20]. The [4×4] matrix $\boldsymbol{R}$ includes weights on control input $\boldsymbol{u}(t)$ and is chosen as identity matrix. The control input (4) is calculated by

$$
\boldsymbol{u}(t)=-\boldsymbol{K}_{\mathrm{LQR}}\left(F_{x}\right) \boldsymbol{x}(t),
$$

where the $[4 \times 12]$ control matrix $\boldsymbol{K}_{\mathrm{LQR}}\left(F_{x}\right)$ is given by

$$
\boldsymbol{K}_{\mathrm{LQR}}\left(F_{x}\right)=\boldsymbol{R}^{-1} \boldsymbol{B}^{T} \boldsymbol{P}\left(F_{x}\right) .
$$

In $(28), \boldsymbol{P}\left(F_{x}\right)$ is the solution of the continuous-time Algebraic RICCATI Equation $(\mathrm{CARE})$

$$
\boldsymbol{A}\left(F_{x}\right)^{T} \boldsymbol{P}\left(F_{x}\right)+\boldsymbol{P}\left(F_{x}\right) \boldsymbol{A}\left(F_{x}\right)-\boldsymbol{P}\left(F_{x}\right) \boldsymbol{B} \boldsymbol{R}^{-1} \boldsymbol{B}^{T} \boldsymbol{P}\left(F_{x}\right)+\boldsymbol{Q}=0
$$

with system matrix $\boldsymbol{A}\left(F_{x}\right)$, input matrix $\boldsymbol{B},(25)$, and weights $\boldsymbol{Q}$ and $\boldsymbol{R}$, [19]. Since the system matrix is a function of axial load $F_{x}$, the solution of $(29)$ and the optimal control matrix (28) change for each different loading. In the experiment, this problem is solved by manually switching between different control matrices which are calculated for axial loads from $0-3500 \mathrm{~N}$ in steps of $500 \mathrm{~N}$ for the quasi-statically increasing axial load. 


\section{Experimental buckling control}

The surface strains according to (5) are measured with strain gauge sensors on the beamcolumn, figure 1. The amplified signal which is filtered by analogue elliptic lowpass anti-aliasing filters is fed into a real-time dSPACE system. The deformation of the beam-column in $y$ - and $z$-direction is approximated by the modal displacements (13) that, due to the invertibility of the $[4 \times 4]$ matrix $\left(\boldsymbol{C}_{0} \boldsymbol{\Phi}\right)$, result in

$$
\boldsymbol{q}_{\mathrm{m}}(t)=\left(\boldsymbol{C}_{0} \boldsymbol{\Phi}\right)^{-1} \boldsymbol{y}(t) .
$$

A first order digital ButTERWORTH highpass filter is used to approximate the modal velocities $\dot{\boldsymbol{q}}_{\mathrm{m}}(t)$ and a discrete integrator sums up the modal displacements to get the integral state vector (21). The state vector (22) is used to calculate the control forces $\boldsymbol{u}(t)$ via $(27)$. The control matrix $\boldsymbol{K}_{\mathrm{LQR}}\left(F_{x}\right)$ is switched manually in steps of $500 \mathrm{~N}$ for the quasi-statically increasing axial load. The control forces are transformed into the directions of the piezoelectric stack actuators, figure 1b. The resulting dSPACE output voltages are filtered by analogue elliptic lowpass reconstruction filters before being amplified and applied to the piezoelectric stack actuator in supports A and B.

The results of the beam-column system with and without active buckling control are shown in figure 7 . The absolute modal displacements of the first modes $q_{1, y}$ and $q_{1, z}$ in $y$ - and $z$-direction are plotted with respect to the axial load $F_{x}$ in figure $7 \mathrm{a}$. Figure $7 \mathrm{~b}$ shows the modal displacements $q_{1, y}$ and $q_{1, z}$ in the $y$-z-plane.

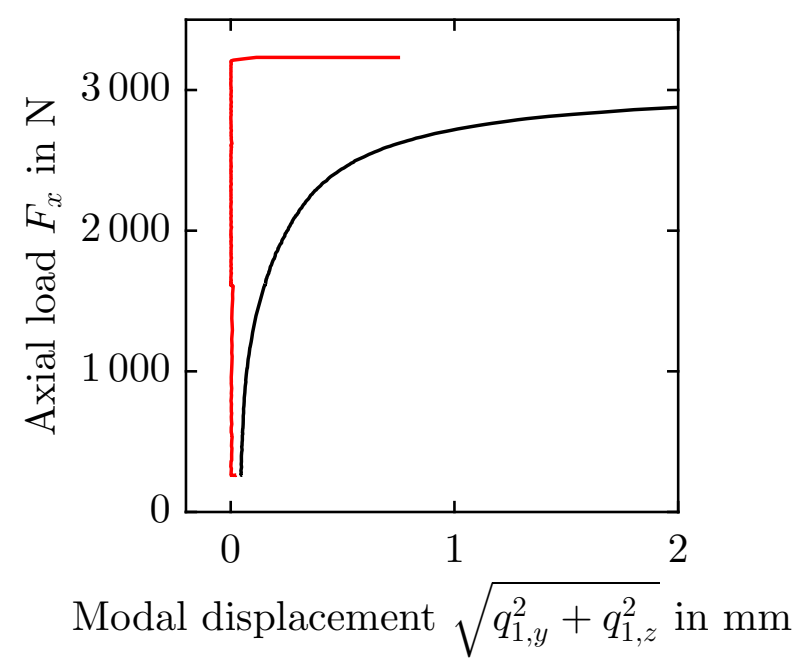

(a)

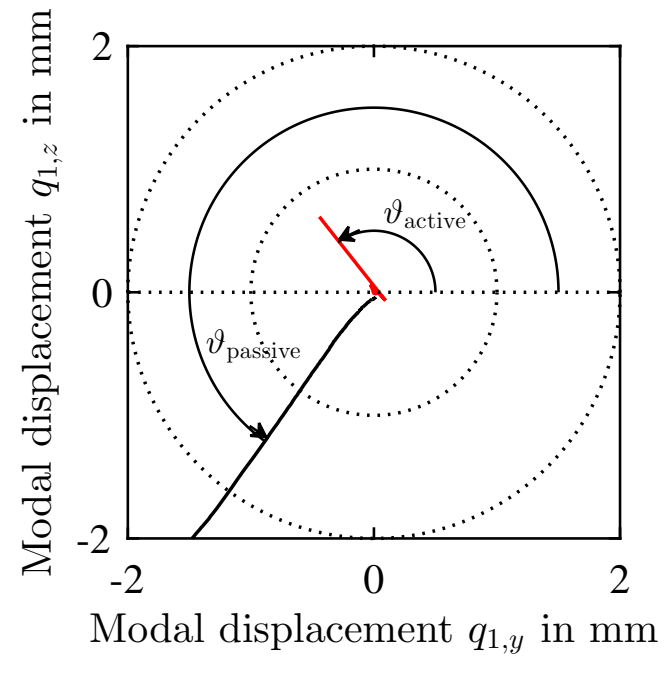

(b)

Figure 7: Modal displacements of the first modes $q_{1, y}$ and $q_{1, z}$ in $y$ - and $z$-direction for the beam-column with (-) and without (-) active buckling control, (a) absolute modal displacement versus axial load $F_{x}$ (b) modal displacements in $y$-z-plane.

Without active buckling control, the load-displacement curve shows the typical continuous deformation with increasing axial load which is known from real, imperfect 
beam-columns, [1]. Sudden buckling does not occur. In theory, the ideal beamcolumn with circular cross-section has infinite directions of buckling. The real beamcolumn, however, continuously deforms in an angle of $\vartheta_{\text {passive }}=234^{\circ}$ measured from the positive $y$-direction. The direction of buckling of the real uncontrolled system is mainly dependent on the assembly conditions of the test setup and varies accordingly. It is not possible to detect one single critical buckling load of the uncontrolled system, only a maximum admissible deformation can be defined depending on the intended application. Maximum deformations of $0.5 \mathrm{~mm}$ and $1 \mathrm{~mm}$ are reached for axial loads of $F_{x, 0.5}=2438 \mathrm{~N}=0.76 F_{x, c r}$ and $F_{x, 1.0}=2723 \mathrm{~N}=0.85 F_{x, c r}$ which is considerably less than the theoretical critical buckling load $F_{x, c r}=3194 \mathrm{~N}$ determined from the FE model via (12).

With active buckling control, the piezoelectric stack actuators in the piezo-elastic supports are able to initially force the beam-column into a straight position and then to reduce the beam-column deformation for the quasi-statically increasing axial load $F_{x}$. The integral LQR is able to stabilize the beam-column to an axial load of $F_{x, \max }=3223 \mathrm{~N}=1.01 F_{x, c r}$. Until $F_{x, \max }$, the deformation in both $y$ - and $z$-direction

remains zero, figure $7 \mathrm{~b}$. At $F_{x, \max }=3223 \mathrm{~N}$, the maximum voltage of $150 \mathrm{~V}$ of the first set of piezoelectric actuators is reached so that the required control forces (4) cannot be provided any more. The beam-column starts to oscillate and finally buckles at an angle of $\vartheta_{\text {active }}=128^{\circ}$. This direction is almost perpendicular to the original direction of buckling observed without active buckling control. This shows that the other piezoelectric stack actuators that have not yet reached their limits continuously try to stabilize the beam-column.

\section{Conclusions}

A new method of active buckling control of an axially loaded beam-column with circular cross-section by piezo-elastic supports is presented and investigated experimentally. With the piezo-elastic supports, lateral forces of piezoelectric stack actuators are transformed into bending moments acting in arbitrary directions at the beam-column ends. A FE model of the beam-column system is derived and validated by an experimental system identification. The model is reduced by modal truncation and augmented by integrated states to implement an integral linear quadratic regulator (LQR). The experiments show that the axially loaded beam-column with circular crosssection without active buckling control exhibits high lateral deformations for axial loads considerably below the theoretical critical buckling load. With active buckling control using the presented piezo-elastic supports, stabilization of the beam-column in arbitrary direction is possible. The integral LQR is able to stabilize the beamcolumn for axial loads up to the theoretical critical buckling load of the beam-column. In future investigations, the modeling of imperfections for an advanced and more complex representation of the beam-column system will be performed. Furthermore, gain scheduled integral LQR control will be implemented and tested with piezoelectric 
stack transducers with the ability to execute higher forces.

\section{Acknowledgments}

The authors like to thank the German Research Foundation (DFG) for funding this project within the Collaborative Research Center (SFB) 805.

\section{References}

[1] Timoshenko, S. P.; Gere, J. M.: Theory of Elastic Stability. McGraw-Hill, New York, 1961.

[2] Meressi, T. ; Paden, B.: Buckling control of a flexible beam using piezoelectric actuators. Journal of Guidance, Control, and Dynamics, 16 (5): 977-980, 1993.

[3] Wang, Q. S.: Active buckling control of beams using piezoelectric actuators and strain gauge sensors. Smart Materials and Structures, 19: 1-8, 2010.

[4] Thompson, S. P. ; Loughlan, J.: The active buckling control of some composite column strips using piezoceramic actuators. Composite Structures, 32: 59-67, 1995.

[5] Berlin, A. A. ; Chase, J. G.; Yim, M. ; Maclean, J. B. ; Olivier, M. ; Jacobsen, S. C.: Mems-based control of structural dynamic instability. Journal of Intelligent Material Systems and Structures, 9: 574- 586, 1998.

[6] Zenz, G. ; Humer, A.: Stability enhancement of beam-type structures by piezoelectric transducers: theoretical, numerical and experimental investigations. Acta Mechanica, 226: 3961-3976, 2015.

[7] Choi, S. ; Lee, J. J. ; Seo, D. C. ; Choi, S. W.: The active buckling control of laminated composite beams with embedded shape memory alloy wires. Composite Structures, 47: 679-686, 1999.

[8] Enss, G. C. ; Platz, R. ; Hanselka, H.: Uncertainty in loading and control of an active column critical to buckling. Shock and Vibration, 19: 929-937, 2012.

[9] Enss, G. C. ; Platz, R.: Statistical approach for active buckling control with uncertainty. In J. De Clerck, editor, Topics in Modal Analysis I, Volume 7: Proceedings of the 32nd IMAC, A Conference and Exposition on Structural Dynamics, 2014, number 209, pages pp. 291-297. Springer International Publishing, (Orlando) 2014.

[10] Schaeffner, M. ; Enss, G. C. ; Platz, R.: Mathematical modeling and numerical simulation of an actively stabilized beam-column with circular cross-section. In Proc. SPIE volume 905\%, (San Diego) 2014.

[11] Schaeffner, M. ; Platz, R. ; Melz, T.: Active buckling control of an axially loaded beamcolumn with circular cross-section by active supports with integrated piezoelectric actuators. In Proceedings of SMART2015 7th ECCOMAS Thematic Conference on Smart Structures and Materials, (Ponta Delgada), 2015.

[12] Götz, B. ; Schaeffner, M. ; Platz, R. ; Melz, T.: Model verification and validation of a piezoelastic support for passive and active structural state control of beams with circular cross-section. Applied Mechanics and Materials, Trans Tech Publications, 807:67-77, 2015.

[13] Klein, B.: FEM. Springer Vieweg, Wiesbaden, 2012.

[14] Przemieniecki, J. S.: Theory of Matrix Structural Analysis. McGraw-Hill, New York, 1968.

[15] Khot, S. M. ; Yelve, N. P.: Modeling and response analysis of dynamic systems by using ANSYS and MATLAB. Journal of Vibration and Control, 0 (0): 1-6, 2010.

[16] Fuller, C. R. ; Elliot, S.J. ; Nelson, P.A.: Active Control of Vibration. Academic Press, Inc., 1997.

[17] Gawronski, W.: Advanced Structural Dynamic and Active Control of Structures. Springer, 2010.

[18] Hendricks, E. ; Jannerup, O. ; Sørensen, P. H.: Linear Systems Control. Springer, 2008.

[19] Skogestad, S. ; Postlethwaite, I.: Multivariable Feedback Control: Analysis and Design. John Wiley \& Sons, New York, 2005.

[20] Lunze, J.: Regelungstechnik 2 Mehrgrößensysteme, Digitale Regelung. Springer-Verlag Berlin Heidelberg, 2008. 